Contents List available at RAZI Publishing

Matriks Sains Matematik (MSMK)

Journal Homepage: http://www.razipublishing.com/journals/matriks-sains-matematik

https://doi.org/10.26480/msmk.02.2017.06.10

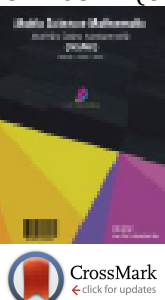

\title{
MULTIGRID SOLUTION FOR THE CAUCHY PROBLEM ASSOCIATED WITH HELMHOLTZ TYPE EQUATION ON NON-UNIFORM GRIDS
}

\author{
Fazal Ghaffar ${ }^{*}$, Noor Badshah ${ }^{2}$, S. Islam ${ }^{1}$ \\ 1 Department of Mathematics Abdul Wali Khan University Mardan, Khyber Pakhtunkhwa, Pakistan. \\ ${ }^{2}$ Department of Basic Sciences University of Engineering and Technology Peshawar, Khyber Pakhtunkhwa, Pakistan. \\ *Corresponding Author email: fghaffarmaths@gmail.com
}

\begin{abstract}
This is an open access article distributed under the Creative Commons Attribution License, which permits unrestricted use, distribution, and reproduction in any medium, provided the original work is properly cited
\end{abstract}

\section{ARTICLE DETAILS}

\section{ARTICLE HISTORY:}

Received 4 July 2017

Accepted 7 October 2017

Available online 3 November 2017

\section{KEYWORDS}

Helmholtz equation, Compact iterative schemes, Multigrid method, Cauchy problem Nonuniform grids.

\section{ABSTRACT}

In this paper, an HOC scheme with multigrid algorithm is developed for solving the Cauchy problem associated with two dimensional Helmholtz type equations. The suggested scheme has up to fourth order accuracy. Lastly, some numerical experiments are given to show the accuracy and performance of the proposed scheme.

\section{INTRODUCTION}

Helmholtz equation has many real-world applications related to wave propagation and vibrating phenomena [1], the radiation and scattering of wave $[2,3]$. We focus on the important application of Helmholtz equation that is the problem of heat conduction in fins [4-6]. The boundary conditions are often incomplete in many engineering problems and the solution is prescribed at some interior points in the domain. Generally, these are called ill-posed problems such that the stability and uniqueness of their solution are not guaranteed [7]. A classic example of an inverse problem for Helmholtz-type equation is the Cauchy problem. In this problem, the boundary conditions for both the solution and its normal derivative are prescribed only on a part of the boundary of the solution domain while having no information about the remaining part of the boundary. The uniqueness of the Cauchy problem is guaranteed unlike the direct problems without removing the eigenvalues for the Laplacian. Most models of real life problems are still very difficult to solve either theoretically or numerically. There has recently devoted to the search for better and more efficient methods for determining an approximate or numerical solution.

The objective of this work is to establish an algorithm based on Multigrid method for Helmholtz equation. Higher order compact schemes (HOC) are used for the solution of Helmholtz equation and other elliptic PDEs [8, 9]. Consider the three-dimensional (3D) Helmholtz equation

$$
u_{x x}+u_{y y}+u_{z z}+\ell^{2} u=f(x, y, z), \quad(x, y, z) \in \Omega
$$

where $\Omega$ is a cubic domain and $\ell$ is a wave number? The forcing function $f(x, y, z)$ and the solution $u(x, y, z)$ have the required continuous differentiability up to a specific order. Helmholtz equation has many real-world applications like elasticity, electromagnetic waves, acoustic wave scattering, weather and climate prediction, water wave propagation, noise reduction in silencers and radar scattering. In this paper, we use a finite difference approximation on non-uniform grids in discrete domain to obtain a scheme up to fourth order accuracy. We also considered Helmholtz equation with constant value of $k$. The discretized form of Equation (1) is

$$
\delta_{x}^{2} u_{i, j, k}+\delta_{y}^{2} u_{i, j, k}+\delta_{z}^{2} u_{i, j, k}+\ell^{2} u_{i, j, k}=f_{i, j, k}+O\left(h^{2}\right)
$$

Equation (1) has been solved by different techniques such as finitedifference method (FDM) [10], fast Fourier transform- based (FFT) methods [11], finite element method (FEM) [12], the spectral-element method [13], compact finite-difference method [14] and multigrid methods [15]. Multigrid method based on HOC schemes is among the most efficient iterative technique for solving PDEs [16, 17].

In FDM the number of mesh points will be enlarged to increase the accuracy however, it will also increase the computational time. Helmholtz equation is solved by FEM and spectral-element method, but the limitations of these methods are of high computational cost [13]. Many iterative techniques for Helmholtz equation suffer due to their slow convergence. The investigation for fast iterative methods to achieve higher order accuracy for PDEs is more attractive a area of research.

Multigrid method together with the HOC schemes on uniform grids are developed in $[18-20,17]$. In most cases, when sudden changes occur in a flow, the step sizes have to be rectified over the entire domain. Under these situations, where points are concentrated in the regions of sharp variation local mesh refinement procedures $[8,15,21-24]$ are necessary, thus dramatically reducing the computational time and computer storage. Cao and Ge developed a multigrid method with HOC scheme on non-uniform grids for solving 2D convection diffusion equation [21]. This paper is based on approach that an interpolation operator and a projection operator that are suited for HOC scheme using non-uniform mesh is represented by transformation-free HOC scheme on non-uniform grids. The main focus in this paper is to develop multigrid method based on HOC scheme on non-uniform grids for solving of 3D Helmholtz equation. To the best of our knowledge 3D Helmholtz equation is not solved by multigrid method based on HOC scheme on non-uniform grids. 


\section{MATHEMATICAL FORMULATION}

For the sake of explanation physically referring to the heat transfer, it is assuming that the temperature field $T(\underline{x})$ satisfies the Helmholtz equation in an open bounded domain $\Omega \in \mathfrak{R}^{d}$, where $d$ is the dimension of the space and $d \in\{1,2,3\}$.

$$
L T(\underline{x})=\left(\Delta+k^{2}\right) T(\underline{x})=\left(\sum_{i=1}^{d} a_{i}^{2}+k^{2}\right) T(\underline{x})=0, x \in \Omega .
$$

where $k=\alpha+i \beta \in C, i=\sqrt{-1}$ and $k^{2} \in \mathfrak{R}$, when $\alpha=0$ and $b \in \mathfrak{R}$, the partial differential Equation (3) models the heat conduction in a fin [4, 5, 6]. Where $T$ is the local fin temperature, $\beta^{2}=\frac{h}{\tilde{k} t}$, such that $\tilde{k}$ is the thermal conductivity, $h$ is the coefficient of the heat transfer of the fin and $t$ is the half- fin thickness. The Cauchy problem under consideration requires solution of PDE (3).

Consider a cubic domain $(x, y, z) \in\left[a_{1}, a_{2}\right] \times\left[b_{1}, b_{2}\right] \times\left[c_{1}, c_{2}\right]$. Discretization is performed on a three-dimensional non-uniform girds points. The above intervals $\left[a_{1}, a_{2}\right],\left[b_{1}, b_{2}\right]$ and $\left[c_{1}, c_{2}\right]$ are divide into subintervals

$$
\begin{aligned}
& a_{1}=x_{0}, x_{1}, x_{2}, \ldots, x_{N_{x}}=a_{2}, \\
& b_{1}=y_{0}, y_{1}, y_{2}, \ldots, y_{N_{y}}=b_{2} \\
& c_{1}=z_{0}, z_{1}, z_{2}, \ldots, z_{N_{z}}=c_{2} .
\end{aligned}
$$

In $x$-direction, consider $h_{x}=\frac{a_{2}-a_{1}}{N_{x}}$, the forward and backward step sizes are gives by

$$
h_{f x}=x_{i+1}-x_{i}=\theta_{f x} h_{x}, \quad h_{b x}=x_{i}-x_{i-1}=\theta_{b x} h_{x}, \quad 1 \leq i<N_{x}-1 .
$$

Also for $y$-direction, $h_{y}=\frac{b_{2}-b_{1}}{N_{y}}$,

$$
h_{f y}=y_{j+1}-y_{j}=\theta_{f y} h_{y}, h_{b y}=y_{j}-y_{j-1}=\theta_{b y} h_{y}, 1 \leq j<N_{y}-1 .
$$

Furthermore, we define $\alpha_{x}, \beta_{x}, \gamma_{x}$ as $\alpha_{x}=\theta_{f x} \theta_{b x}, \quad \beta_{x}=\theta_{f x}+\theta_{b x}$ and $\gamma_{x}=\theta_{f x}-\theta_{f x}$.

Similarly $\alpha_{y}, \beta_{y}, \gamma_{y}$ and $\alpha_{z}, \beta_{z}, \gamma_{z}$ can be find accordingly for $y-$ and $z$-directions. If $\theta_{f x}=\theta_{b x}=1\left(h_{f x}=h_{b x}, h_{f y}=h_{b y}, h_{f z}=h_{b z}\right)$ then the grids turn to be uniform. The approximate value of a function $u(x, y, z)$ at the interior grids points $\left(x_{i}, y_{j}, z_{k}\right)$ is represented by $u_{0}$ and the approximate values of other twenty six (26) nearest grid points are determined by $u_{i}$ , $i=1,2,3, \ldots, 26$, as in Figure $1(\mathrm{~b})$
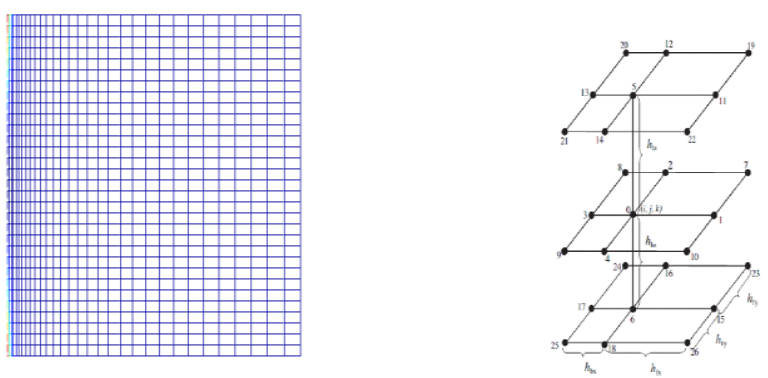

Fig. 1: (a) Nonuniform grids distribution in xy-plane $\quad$ (b) Stencil of nonuniform 3D grids Taylor series expansion is performed for appropriate description of sufficient smooth function $u(x, y, z)$ in the given domain at points 1 and 3 are,

$$
\begin{aligned}
u_{1}= & u_{0}+\theta_{f x} h_{x} \partial_{x} u_{0}+\frac{\theta_{f x}^{2} h_{x}^{2}}{2} \partial_{x}^{2} u_{0}+\frac{\theta_{f x}^{3} h_{x}^{3}}{6} \partial_{x}^{3} u_{0}+\frac{\theta_{f x}^{4} h_{x}^{4}}{24} \partial_{x}^{4} u_{0} \\
& +\frac{\theta_{f x}^{5} h_{x}^{5}}{120} \partial_{x}^{5} u_{0}+O\left(\theta_{f x}^{6} h_{x}^{6}\right),
\end{aligned}
$$

$$
\begin{aligned}
u_{3}= & u_{0}-\theta_{b x} h_{x} \partial_{x} u_{0}+\frac{\theta_{b x}^{2} h_{x}^{2}}{2} \partial_{x}^{2} u_{0}-\frac{\theta_{b x}^{3} h_{x}^{3}}{6} \partial_{x}^{3} u_{0}+\frac{\theta_{b x}^{4} h_{x}^{4}}{24} \partial_{x}^{4} u_{0} \\
& -\frac{\theta_{b x}^{5} h_{x}^{5}}{120} \partial_{x}^{5} u_{0}+O\left(\theta_{b x}^{6} h_{x}^{6}\right) .
\end{aligned}
$$

Multiplying equation (4) by $\theta_{b x}$ and equation (5) by $\theta_{f x}$, then adding and solving for the second derivative which gives

$$
\begin{aligned}
\partial_{x}^{2} u_{0}= & \frac{2}{\alpha_{x} \beta_{x} h_{x}^{2}}\left(\theta_{b x} u_{1}-\beta_{x} u_{0}+\theta_{f x} u_{3}\right)-\frac{h_{x}}{3} \gamma_{x} \partial_{x}^{3} u_{0} \\
& -\frac{h_{x}^{2}}{12}\left(\beta_{x}^{2}-3 \alpha_{x}\right) \partial_{x}^{4} u_{0}-\frac{h_{x}^{3}}{60}\left(\beta_{x}^{2}-2 \alpha_{x}\right) \gamma_{x} \partial_{x}^{5} u_{0} \\
& +O\left(\eta_{x} h_{x}^{4}\right),
\end{aligned}
$$

in equation (6) $\eta_{x}=\beta_{x}^{4}-5 \alpha_{x}\left(\beta_{x}^{2}-\alpha_{x}\right)$, hence the second order central difference operator along $x$-direction is defined as

$$
\delta_{x}^{2} u_{0}=\frac{2}{\alpha_{x} \beta_{x} h_{x}^{2}}\left(\theta_{b x} u_{1}-\beta_{x} u_{0}+\theta_{f_{x}} u_{3}\right),
$$

if $\theta_{f x}=\theta_{b x}=1$, equation (7) reduces to uniform grids of central difference operator. Therefore, the second order derivative for $x$ - direction is

$$
\begin{aligned}
\partial_{x}^{2} u_{0}= & \delta_{x}^{2} u_{0}-\frac{h_{x}}{3} \gamma_{x} \partial_{x}^{3} u_{0}-\frac{h_{x}^{2}}{12}\left(\beta_{x}^{2}-3 \alpha_{x}\right) \partial_{x}^{4} u_{0} \\
& -\frac{h_{x}^{3}}{60}\left(\beta_{x}^{2}-2 \alpha_{x}\right) \gamma_{x} \partial_{x}^{5} u_{0}+O\left(\eta_{x} h_{x}^{4}\right),
\end{aligned}
$$

the approximation of the second order derivative for the variables $y$ and $z$ can be find accordingly. Therefore, the central difference scheme for Helmholtz equation can be discretized as

$$
\delta_{x}^{2} u_{0}+\delta_{y}^{2} u_{0}+\delta_{z}^{2} u_{0}+\ell^{2}\left(u_{0}\right)=f_{0}+\tau_{0} .
$$

Where $\tau_{0}$ is the truncation error and is defined as?

$$
\begin{aligned}
\tau_{0}= & H_{1} \frac{\partial^{3} u_{0}}{\partial x^{3}}+K_{1} \frac{\partial^{3} u_{0}}{\partial y^{3}}+L_{1} \frac{\partial^{3} u_{0}}{\partial z^{3}}+H_{2} \frac{\partial^{4} u_{0}}{\partial x^{4}}+K_{2} \frac{\partial^{4} u_{0}}{\partial y^{4}} \\
& +L_{2} \frac{\partial^{4} u_{0}}{\partial z^{4}}+H_{3} \frac{\partial^{5} u_{0}}{\partial x^{5}}+K_{3} \frac{\partial^{5} u_{0}}{\partial y^{5}}+L_{2} \frac{\partial^{5} u_{0}}{\partial z^{5}} \\
& +O\left(\eta_{x} h_{x}^{4}\right)+O\left(\eta_{y} h_{y}^{4}\right)+O\left(\eta_{x} z h_{z}^{4}\right) .
\end{aligned}
$$

$H_{1}, H_{2}, H_{3}, L_{1}, L_{2}, L_{3}$ and $K_{1}, K_{2}, K_{3}$ are defined as

$$
\begin{aligned}
& H_{1}=\frac{1}{3} h_{x} \gamma_{x}, \quad K_{1}=\frac{1}{3} h_{y} \gamma_{y}, \quad L_{1}=\frac{1}{3} h_{z} \gamma_{z}, \\
& H_{2}=\frac{1}{12} h_{x}^{2}\left(\beta_{x}^{2}-3 \alpha_{x}\right), \quad K_{2}=\frac{1}{12} h_{y}^{2}\left(\beta_{y}^{2}-3 \alpha_{y}\right), \\
& K_{3}=\frac{1}{60} h_{y}^{3}\left(\beta_{y}^{2}-2 \alpha_{y}\right) \gamma_{y}, \quad L_{2}=\frac{1}{12} h_{z}^{2}\left(\beta_{z}^{2}-3 \alpha_{z}\right), \\
& H_{3}=\frac{1}{60} h_{x}^{3}\left(\beta_{x}^{2}-2 \alpha_{x}\right) \gamma_{x}, \\
& L_{3}=\frac{1}{60} h_{z}^{3}\left(\beta_{z}^{2}-2 \alpha_{z}\right) \gamma_{z}, \eta_{y}=\beta_{y}^{4}-5 \alpha_{y}\left(\beta_{y}^{2}-\alpha_{y}\right), \\
& \eta_{z}=\beta_{z}^{4}-5 \alpha_{z}\left(\beta_{z}^{2}-\alpha_{z}\right) .
\end{aligned}
$$

If $\tau_{0}$ is dropped off from Equation (9), the central difference scheme (CDS) for non-uniform grids will be

$$
\delta_{x}^{2} u_{0}+\delta_{y}^{2} u_{0}+\delta_{z}^{2} u_{0}+\ell^{2}\left(u_{0}\right)=f_{0} .
$$

According to the definition of $\delta_{x}^{2}, \delta_{y}^{2}$ and $\delta_{z}^{2}$ the central difference scheme can be written as

$$
\begin{aligned}
& -2\left(\frac{1}{\alpha_{x} h_{x}^{2}}+\frac{1}{\alpha_{y} h_{y}^{2}}+\frac{1}{\alpha_{z} h_{z}^{2}}\right) u_{0}+\frac{2 \theta_{b x} u_{1}}{\alpha_{x} \beta_{x} h_{x}^{2}}+\frac{2 \theta_{b y} u_{2}}{\alpha_{y} \beta_{y} h_{y}^{2}} \\
& +\frac{2 \theta_{f x} u_{3}}{\alpha_{x} \beta_{x} h_{x}^{2}}+\frac{2 \theta_{f y} u_{4}}{\alpha_{y} \beta_{y} h_{y}^{2}}+\frac{2 \theta_{b z} u_{5}}{\alpha_{z} \beta_{z} h_{z}^{2}}+\frac{2 \theta_{f z} u_{6}}{\alpha_{z} \beta_{z} h_{z}^{2}}=f_{0} .
\end{aligned}
$$

In above Equation (12) only seven grids points are involved. From the 
definition of $\tau_{0}$, it is observed that when $h_{f x}=h_{b x}, h_{f y}=h_{b y}$ and $h_{f z}=h_{b z}$, then Equation (12) is of third order accuracy. In order to improve the order of accuracy we consider

$$
\begin{aligned}
& H_{1} \frac{\partial^{3} u_{0}}{\partial x^{3}}+K_{1} \frac{\partial^{3} u_{0}}{\partial y^{3}}+L_{1} \frac{\partial^{3} u_{0}}{\partial z^{3}}=H_{1} \frac{\partial}{\partial x}+K_{1} \frac{\partial}{\partial y}+L_{1} \frac{\partial}{\partial z} \frac{\partial^{2} u_{0}}{\partial x^{2}}+\frac{\partial^{2} u_{0}}{\partial y^{2}}+\frac{\partial^{2} u_{0}}{\partial z^{2}} \\
&-H_{1} \frac{\partial^{3} u_{0}}{\partial x \partial y^{2}}+H_{1} \frac{\partial^{3} u_{0}}{\partial x \partial z^{2}}+K_{1} \frac{\partial^{3} u_{0}}{\partial y \partial x^{2}}+K_{1} \frac{\partial^{3} u_{0}}{\partial y \partial z^{2}}+L_{1} \frac{\partial^{3} u_{0}}{\partial z \partial y^{2}}+L_{1} \frac{\partial^{3} u_{0}}{\partial z \partial x^{2}} \\
&=H_{1} \frac{\partial f_{0}}{\partial x}+K_{1} \frac{\partial f_{0}}{\partial y}+L_{1} \frac{\partial f_{0}}{\partial z}-l^{2} H_{1} \frac{\partial u_{0}}{\partial x}+K_{1} \frac{\partial u_{0}}{\partial y}+L_{1} \frac{\partial u_{0}}{\partial z} \\
&-H_{1} \frac{2}{\alpha_{y} \beta_{y} h_{y}^{2}} \theta_{b y} \frac{\partial u_{2}}{\partial x}-\beta_{y} \frac{\partial u_{0}}{\partial x}+\theta_{f y} \frac{\partial u_{4}}{\partial x}+O\left(h_{y}^{2}\right) \\
&-H_{1} \frac{2}{\alpha_{z} \beta_{z} h_{z}^{2}} \theta_{b z} \frac{\partial u_{5}}{\partial x}-\beta_{z} \frac{\partial u_{0}}{\partial x}+\theta_{f z} \frac{\partial u_{6}}{\partial x}+O\left(h_{z}^{2}\right) \\
&-K_{1} \frac{2}{\alpha_{x} \beta_{x} h_{x}^{2}} \theta_{b x} \frac{\partial u_{1}}{\partial y}-\beta_{x} \frac{\partial u_{0}}{\partial y}+\theta_{f x} \frac{\partial u_{3}}{\partial y}+O\left(h_{x}^{2}\right) \\
&-K_{1} \frac{2}{\alpha_{z} \beta_{z} h_{z}^{2}} \theta_{b z} \frac{\partial u_{5}}{\partial y}-\beta_{z} \frac{\partial u_{0}}{\partial y}+\theta_{f z} \frac{\partial u_{6}}{\partial y}+O\left(h_{z}^{2}\right) \\
&-L_{1} \frac{2}{\alpha_{x} \beta_{x} h_{x}^{2}} \theta_{b x} \frac{\partial u_{1}}{\partial z}-\beta_{x} \frac{\partial u_{0}}{\partial z}+\theta_{f x} \frac{\partial u_{3}}{\partial z}+O\left(h_{x}^{2}\right) \\
&-L_{1} \frac{2}{\alpha_{y} \beta_{y} h_{y}^{2}} \theta_{b y} \frac{\partial u_{2}}{\partial z}-\beta_{y} \frac{\partial u_{0}}{\partial z}+\theta_{f y} \frac{\partial u_{4}}{\partial z}+O\left(h_{y}^{2}\right),
\end{aligned}
$$

and

$$
\begin{aligned}
& H_{2} \frac{\partial^{4} u_{0}}{\partial x^{2}}+K_{\frac{1}{2}} \frac{\partial^{4} u_{0}}{\partial y^{4}}+L_{2} \frac{\partial^{4} u_{0}}{\partial z^{2}}=\left(H_{2} \frac{\partial^{2}}{\partial x^{2}}+K_{2} \frac{\partial^{2}}{\partial y^{2}}+L_{2} \frac{\partial^{2}}{\partial z^{2}}\right)\left(\frac{\partial^{2} u_{0}}{\partial x^{2}}+\frac{\partial^{2} u_{0}}{\partial y^{2}}+\frac{\partial^{2} u_{0}}{\partial z^{2}}\right) \\
& -\left[\left(H_{2}+K_{2}\right) \frac{\partial^{2} u_{0}}{\partial x^{2} \partial y^{2}}+\left(H_{2}+L_{2}\right) \frac{\partial^{2} u_{0^{2}}}{\partial x^{2} \partial z^{2}}+\left(K_{2}+L_{2}\right) \frac{\partial^{2} u_{0^{2}}}{\partial y^{2} \partial z^{2}}\right]
\end{aligned}
$$

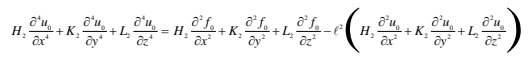

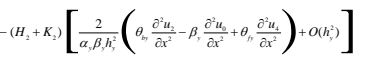

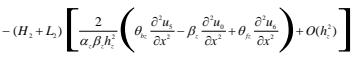

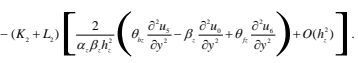

(14)

Through central difference schemes the first order and second order derivative in Equations (13) and (14) can be approximated. Now combining equations (9) and (10) with equations (13) and (14), the nineteen point HOC scheme on non-uniform mesh points for the threedimensional Helmholtz Equation (1) can be written as

$$
\begin{aligned}
\sum_{i=0}^{18} A_{i} u_{i} & =f_{0}+H_{1} \frac{\partial f_{0}}{\partial x}+K_{1} \frac{\partial f_{0}}{\partial y}+L_{1} \frac{\partial f_{0}}{\partial z}+H_{2} \frac{\partial^{2} f_{0}}{\partial x^{2}} \\
& +K_{2} \frac{\partial^{2} f_{0}}{\partial x^{2}}+L_{2} \frac{\partial^{2} f_{0}}{\partial z^{2}} .
\end{aligned}
$$

The coefficients on the LHS in Equation (15) are given as

$$
\begin{aligned}
& A_{0}=-2 \frac{1}{\alpha_{x} h_{x}^{2}}+\frac{1}{\alpha_{y} h_{y}^{2}}+\frac{1}{\alpha_{z} h_{z}^{2}}-2 \frac{l^{2} H_{2}}{\alpha_{x} h_{x}^{2}}+\frac{l^{2} K_{2}}{\alpha_{y} h_{y}^{2}}+\frac{l^{2} L_{2}}{\alpha_{z} h_{z}^{2}}+4 \frac{\left(H_{2}+K_{2}\right)}{\alpha_{x} \alpha_{y} h_{x}^{2} h_{y}^{2}}+\frac{\left(K_{2}+L_{2}\right)}{\alpha_{z} \alpha_{y} h_{z}^{2} h_{y}^{2}}+\frac{\left(H_{2}+L_{2}\right)}{\alpha_{x} \alpha_{z} h_{x}^{2} h_{z}^{2}}, \\
& A_{1}=2 \frac{\theta_{b x}}{\alpha_{x} \beta_{x} h_{x}^{2}}-\frac{H_{1}}{\alpha_{y} \beta_{x} h_{x} h_{y}^{2}}-\frac{H_{1}}{\alpha_{z} \beta_{x} h_{x} h_{z}^{2}}+\frac{l^{2} H_{2} \theta_{b x}}{\alpha_{x} \beta_{x} h_{x}^{2}}+\frac{l^{2} H_{1}}{\beta_{x} h_{x}}-4 \frac{\left(H_{2}+K_{2}\right) \theta_{b x}}{\alpha_{x} \alpha_{y} \beta_{x} h_{x}^{2} h_{y}^{2}}+\frac{\left(H_{2}+L_{2}\right) \theta_{b x}}{\alpha_{x} \alpha_{z} \beta_{x} h_{x}^{2} h_{z}^{2}}, \\
& A_{2}=2 \frac{\theta_{b y}}{\alpha_{y} \beta_{y} h_{y}^{2}}+\frac{l^{2} K_{2} \theta_{b y}}{\alpha_{y} \beta_{y} h_{y}^{2}}-\frac{K_{1}}{\alpha_{x} \beta_{y} h_{x}^{2} h_{y}}-\frac{K_{1}}{\alpha_{z} \beta_{y} h_{y} h_{z}^{2}}+\frac{\ell^{2} K_{1}}{\beta_{y} h_{y}}-4 \frac{\left(H_{2}+K_{2}\right) \theta_{b y}}{\alpha_{x} \alpha_{y} \beta_{y} h_{x}^{2} h_{y}^{2}}+\frac{\left(K_{2}+L_{2}\right) \theta_{b y}}{\alpha_{z} \alpha_{y} \beta_{y} h_{y}^{2} h_{z}^{2}}, \\
& A_{3}=2 \frac{\theta_{f x}}{\alpha_{x} \beta_{x} h_{x}^{2}}+\frac{\ell^{2} H_{2} \theta_{f x}}{\alpha_{x} \beta_{x} h_{x}^{2}}+\frac{H_{1}}{\alpha_{y} \beta_{x} h_{x} h_{y}^{2}}+\frac{H_{1}}{\alpha_{z} \beta_{x} h_{x} h_{z}^{2}}-\frac{l^{2} H_{1}}{\beta_{x} h_{x}}-4 \frac{\left(H_{2}+K_{2}\right) \theta_{f x}}{\alpha_{x} \alpha_{v} \beta_{x} h_{x}^{2} h_{y}^{2}}+\frac{\left(H_{2}+L_{2}\right) \theta_{f x}}{\alpha_{x} \alpha_{z} \beta_{x} h_{x}^{2} h_{z}^{2}} \\
& A_{4}=2 \frac{\theta_{f y}}{\alpha_{y} \beta_{y} h_{y}^{2}}+\frac{\ell^{2} K_{2} \theta_{f y}}{\alpha_{y} \beta_{y} h_{y}^{2}}+\frac{K_{1}}{\alpha_{2} \beta_{y} h^{2} h_{y}}+\frac{K_{1}}{\alpha_{2} \beta_{y} h_{y} h_{2}^{2}}-\frac{\ell^{2} K_{1}}{\beta_{y} h_{y}}-4 \frac{\left(H_{2}+K_{2}\right) \theta_{f y}}{\alpha_{\alpha} \alpha_{y} \beta_{y} h_{2}^{2} h_{y}^{2}}+\frac{\left(K_{2}+L_{2}\right) \theta_{f y}}{\alpha_{y} \alpha_{2} \beta_{y} h_{2}^{2} h_{2}^{2}}, \\
& A_{5}=2 \frac{\theta_{b z}}{\alpha_{z} \beta_{z} h_{z}^{2}}+\frac{\ell^{2} L_{2} \theta_{b z}}{\alpha_{z} \beta_{z} h_{z}^{2}}-\frac{L_{1}}{\alpha_{x} \beta_{z} h_{x}^{2} h_{z}}-\frac{L_{1}}{\alpha_{z} \beta_{z} h_{y}^{2} h_{z}}+\frac{\ell^{2} L_{1}}{\beta_{z} h_{z}}-4 \frac{\left(K_{2}+L_{2}\right) \theta_{b z}}{\alpha_{y} \alpha_{z} \beta_{z} h_{y}^{2} h_{z}^{2}}+\frac{\left(H_{2}+L_{2}\right) \theta_{b z}}{\alpha_{x} \alpha_{z} \beta_{z} h_{x}^{2} h_{z}^{2}}, \\
& A_{6}=2 \frac{\theta_{f z}}{\alpha_{z} \beta_{z} h_{z}^{2}}+\frac{\ell^{2} L_{2} \theta_{f z}}{\alpha_{z} \beta_{z} h_{z}^{2}}+\frac{L_{1}}{\alpha_{x} \beta_{z} h_{x}^{2} h_{z}}+\frac{L_{1}}{\alpha_{y} \beta_{z} h_{y}^{2} h_{z}}-\frac{\ell^{2} L_{1}}{\beta_{y} h_{y}}-4 \frac{\left(K_{2}+L_{2}\right) \theta_{f z}}{\alpha_{y} \alpha_{z} \beta_{z} h_{y}^{2} h_{z}^{2}}+\frac{\left(H_{2}+L_{2}\right) \theta_{f z}}{\alpha_{x} \alpha_{z} \beta_{z} h_{x}^{2} h_{z}^{2}}, \\
& A_{7}=\frac{2 H_{1} \theta_{b y}}{\alpha_{y} \beta_{y} \beta_{x} h_{x} h_{y}^{2}}+\frac{2 K_{1} \theta_{b x}}{\alpha_{x} \beta_{x} \beta_{y} h_{x}^{2} h_{y}}+\frac{4\left(H_{2}+K_{2}\right) \theta_{b x} \theta_{b y}}{\alpha_{x} \alpha_{y} \beta_{x} \beta_{y} h_{x}^{2} h_{y}^{2}}, \\
& A_{8}=\frac{2 K_{1} \theta_{f x}}{\alpha_{x} \beta_{x} \beta_{y} h_{x}^{2} h_{y}}-\frac{2 H_{1} \theta_{b y}}{\alpha_{y} \beta_{x} \beta_{y} h_{x} h_{y}^{2}}+\frac{4\left(H_{2}+K_{2}\right) \theta_{b y} \theta_{f x}}{\alpha_{x} \alpha_{y} \beta_{x} \beta_{y} h_{x}^{2} h_{y}^{2}}, \\
& A_{9}=\frac{-2 K_{1} \theta_{f x}}{\alpha_{x} \beta_{x} \beta_{y} h_{x}^{2} h_{y}}-\frac{2 H_{1} \theta_{f y}}{\alpha_{y} \beta_{x} \beta_{y} h_{x} h_{y}^{2}}+\frac{4\left(H_{2}+K_{2}\right) \theta_{f x} \theta_{f y}}{\alpha_{x} \alpha_{y} \beta_{x} \beta_{y} h_{x}^{2} h_{y}^{2}} \\
& A_{10}=\frac{2 H_{1} \theta_{f y}}{\alpha_{y} \beta_{x} \beta_{y} h_{x} h_{y}^{2}}-\frac{2 K_{1} \theta_{b x}}{\alpha_{x} \beta_{x} \beta_{y} h_{x}^{2} h_{y}}+\frac{4\left(H_{2}+K_{2}\right) \theta_{b x} \theta_{f y}}{\alpha_{x} \alpha_{y} \beta_{x} \beta_{y} h_{x}^{2} h_{y}^{2}},
\end{aligned}
$$

$$
\begin{aligned}
& A_{11}=\frac{2 H_{1} \theta_{b z}}{\alpha_{z} \beta_{x} \beta_{z} h_{x} h_{z}^{2}}+\frac{2 L_{1} \theta_{b x}}{\alpha_{x} \beta_{x} \beta_{z} h_{x}^{2} h_{z}}+\frac{4\left(H_{2}+L_{2}\right) \theta_{b x} \theta_{b z}}{\alpha_{x} \alpha_{z} \beta_{x} \beta_{z} h_{x}^{2} h_{z}^{2}}, \\
& A_{12}=\frac{2 K_{1} \theta_{b z}}{\alpha_{z} \beta_{y} \beta_{z} h_{y} h_{z}^{2}}+\frac{2 L_{1} \theta_{b y}}{\alpha_{y} \beta_{y} \beta_{z} h_{y}^{2} h_{z}}+\frac{4\left(K_{2}+L_{2}\right) \theta_{b y} \theta_{b z}}{\alpha_{y} \alpha_{z} \beta_{y} \beta_{z} h_{y}^{2} h_{z}^{2}}, \\
& A_{13}=\frac{2 L_{1} \theta_{f x}}{\alpha_{x} \beta_{x} \beta_{z} h_{x}^{2} h_{z}}-\frac{2 H_{1} \theta_{b z}}{\alpha_{z} \beta_{x} \beta_{z} h_{x} h_{z}^{2}}+\frac{4\left(H_{2}+L_{2}\right) \theta_{f x} \theta_{b z}}{\alpha_{x} \alpha_{z} \beta_{x} \beta_{z} h_{x}^{2} h_{z}^{2}}, \\
& A_{14}=\frac{2 L_{1} \theta_{f y}}{\alpha_{y} \beta_{y} \beta_{z} h_{y}^{2} h_{z}}-\frac{2 K_{1} \theta_{b z}}{\alpha_{z} \beta_{y} \beta_{z} h_{y} h_{z}^{2}}+\frac{4\left(K_{2}+L_{2}\right) \theta_{f y} \theta_{b z}}{\alpha_{y} \alpha_{z} \beta_{y} \beta_{z} h_{y}^{2} h_{z}^{2}}, \\
& A_{15}=\frac{2 H_{1} \theta_{f z}}{\alpha_{z} \beta_{x} \beta_{z} h_{x} h_{z}^{2}}-\frac{2 L_{1} \theta_{b x}}{\alpha_{x} \beta_{x} \beta_{z} h_{x}^{2} h_{z}}+\frac{4\left(H_{2}+L_{2}\right) \theta_{b x} \theta_{f z}}{\alpha_{x} \alpha_{z} \beta_{x} \beta_{z} h_{x}^{2} h_{z}^{2}}, \\
& A_{16}=\frac{2 K_{1} \theta_{f z}}{\alpha_{z} \beta_{y} \beta_{z} h_{y} h_{z}^{2}}-\frac{2 L_{1} \theta_{b y}}{\alpha_{y} \beta_{y} \beta_{z} h_{y}^{2} h_{z}}+\frac{4\left(K_{2}+L_{2}\right) \theta_{b y} \theta_{f z}}{\alpha_{y} \alpha_{z} \beta_{y} \beta_{z} h_{y}^{2} h_{z}^{2}}, \\
& A_{17}=-\frac{2 H_{1} \theta_{f z}}{\alpha_{z} \beta_{x} \beta_{z} h_{x} h_{z}^{2}}-\frac{2 L_{1} \theta_{f x}}{\alpha_{x} \beta_{x} \beta_{z} h_{x}^{2} h_{z}}+\frac{4\left(H_{2}+L_{2}\right) \theta_{f x} \theta_{f z}}{\alpha_{x} \alpha_{z} \beta_{x} \beta_{z} h_{x}^{2} h_{z}^{2}}, \\
& A_{18}=-\frac{2 K_{1} \theta_{f z}}{\alpha_{z} \beta_{y} \beta_{z} h_{y} h_{z}^{2}}-\frac{2 L_{1} \theta_{f y}}{\alpha_{y} \beta_{y} \beta_{z} h_{y}^{2} h_{z}}+\frac{4\left(K_{2}+L_{2}\right) \theta_{f y} \theta_{f z}}{\alpha_{y} \alpha_{z} \beta_{y} \beta_{z} h_{y}^{2} h_{z}^{2}} .
\end{aligned}
$$

It is easier to know that this scheme has fourth order of accuracy from expansion of $\tau_{0}$. Under uniform grids distribution the scheme has four to fifth order accuracy as proposed by $[25,26,18]$.

\section{MULTIGRID METHOD}

Multigrid method is one of the most efficient and fastest method for solving PDEs. In multigrid method, the rate of convergence is independent of the mesh size. This method is more effective for solving large scale of sparse linear systems obtained from the discretization of elliptic PDEs [15, 27, 16, 28, 29]. The main principle of multigrid method is to smooth the error on coarse grid level using basic iterative methods such as Jacobi or Gauss-Seidel method, etc. Multigrid method consists of three important components that are relaxation, restriction and interpolation operators. These are applied as "a single iteration of multigrid cycle comprised of manipulating the error by the application of relaxation method, fixing the residuals on the coarse grid level, solving the error equation on the coarse grid and adjusting the correction of coarse grid up to the fine grid level".

Some specific methods have been applied for the solution of 2D and 3D Helmholtz equation with HOC schemes on uniform grids $[8,18,19,12,14$, $10,17,29]$. A full weighting restriction operator and the standard bilinear interpolation operator are used as the inter grid transfer operators. But in case of non-uniform grids these restriction and interpolation operators are quite different

\section{NUMERICAL EXPERIMENTS}

In order to check the effectiveness of the present method, some problems are chosen. V-cycle multigrid method is used with zero initial guess and the process is stopped when the Euclidean norm of the residual vector is reduced by $10^{-10}$ on the finest grid level. The effectiveness of multigrid method with HOC scheme and CD scheme (12) is presented. The reported errors are the maximum absolute errors between the computed solution and the exact solution on finest grid. The order of accuracy for a difference scheme is defined as,

$$
\text { Order }=\log _{2} \frac{\operatorname{Error}\left(N_{1}\right)}{\operatorname{Error}\left(N_{2}\right)},
$$

where $\operatorname{Error}\left(N_{1}\right)$ and $\operatorname{Error}\left(N_{2}\right)$ are maximum absolute errors approximated for two different grids with $N_{1}+1$ and $N_{2}+1$ points in both directions while $N_{1}$ is half of $N_{2}$.

Example 1. Consider the following elliptic PDE with the source term

$$
\begin{aligned}
u_{x x}+u_{y y}+u_{z z}+\ell^{2} u= & f(x, y, z), \\
0<x<1,0<y<1,0<z<1 . & \\
f(x, y, z)= & \left(\ell^{2}+10^{4} e^{-100 x}\right)[y(1-y)-x-z(1-z)] \\
+ & 200 e^{-100 x},
\end{aligned}
$$

the boundary conditions and the source function are given by the analytic solution, that is

$$
u(x, y, z)=e^{-100 x}[y(1-y)-x-z(z-1)] .
$$


The above problem has a steep boundary layer along $x=0$; therefore, we are using non-uniform grids along $x$ - axis which are accumulating near $x=0$ and uniform grids along $y, z$-axis with the following stretching function [29],

$$
x_{i}=\frac{i}{N_{x}}+\frac{\lambda}{\pi} \sin \left(\frac{\pi i}{N_{x}}\right), \quad y_{j}=\frac{j}{N_{y}}, \quad z_{k}=\frac{k}{N_{z}}
$$

where $\lambda$ is a stretching parameter, and controlling the tightness of the grid points in $x$-direction? When $\lambda<0$, then more grid points are accumulated to the boundary $x=0$ and the boundary $x=1$ for $\lambda>0$ .If $\lambda=0$, then the grids reduce to be uniform. Also, when the grid points are $32^{2}$ and $\lambda=-0.8$, then the distribution of grids in $x y$-plane is shown as in Figure 1(a). The estimated accuracy and maximum absolute error with different stretching parameter $\lambda$ is presented in Table 1 . When $\lambda=0$, then the results are very poor. More accurate solution and order of convergence is obtained from HOC schemes with decreasing stretching parameter $\lambda$ in non-uniform grids. We observed that when $\lambda=-0.8$, the solution obtained with HOC schemes is more accurate, but when $\lambda$ is decreases continuously to -0.9 , then the accuracy does not further increase. This situation is not wondering because putting more grids in the boundary layer area that will necessarily cause lack of mesh points in the other regions in domain. Figure 2, indicates the configuration of solution in $x y$-plane with $y=0.5$, (a) shows the exact solution, (b) solution obtained from HOC scheme on uniform grids, (c) HOC scheme non-uniform grids, (d) computed solution with CDS scheme non-uniform grids.

Table 1: Comparison of maximum absolute errors, CPU timing and order of accuracy of the two schemes for Example 1, $\left\|e_{2}\right\|, \ell=10, N=16,32,64,128$

\begin{tabular}{|cc|c|c|c|c|c|c|}
\hline$N$ & $\lambda$ & $16^{2}$ & $32^{2}$ & $64^{2}$ & $128^{2}$ & CPU(seconds) & Order \\
\hline & 0.0 & $7.8052 e^{-1}$ & $6.9881 e^{-1}$ & $4.9012 e^{-1}$ & $5.4880 e^{-2}$ & 0.590 & 1.88 \\
& 0.2 & $5.4212 e^{-1}$ & $1.1012 e^{-1}$ & $7.1100 e^{-2}$ & $6.3876 e^{-3}$ & 1.500 & 1.98 \\
CDS & 0.4 & $1.1184 e^{-1}$ & $7.1572 e^{-2}$ & $3.8067 e^{-2}$ & $1.0695 e^{-3}$ & 1.610 & 1.99 \\
& 0.6 & $7.8100 e^{-2}$ & $3.9180 e^{-2}$ & $5.8733 e^{-3}$ & $8.3434 e^{-4}$ & 1.980 & 2.20 \\
& 0.8 & $4.8327 e^{-2}$ & $6.1500 e^{-3}$ & $1.9150 e^{-3}$ & $1.8422 e^{-4}$ & 2.430 & 2.60 \\
& 0.9 & $8.4346 e^{-3}$ & $6.1984 e^{-3}$ & $6.8769 e^{-4}$ & $8.5500 e^{-5}$ & 3.600 & 3.00 \\
\hline & 0.0 & $4.8322 e^{-1}$ & $3.6630 e^{-2}$ & $4.4090 e^{-2}$ & $7.1218 e^{-4}$ & 0.610 & 2.95 \\
& 0.2 & $16.3118 e^{-2}$ & $9.8861 e^{-3}$ & $6.3039 e^{-3}$ & $8.8812 e^{-5}$ & 0.700 & 3.10 \\
HOC & -0.4 & $7.8711 e^{-2}$ & $2.6821 e^{-3}$ & $2.1025 e^{-3}$ & $1.0080 e^{-5}$ & 1.840 & 3.84 \\
& -0.6 & $3.8237 e^{-2}$ & $6.8582 e^{-3}$ & $8.8101 e^{-4}$ & $6.5230 e^{-6}$ & 2.800 & 3.95 \\
& -0.8 & $8.6227 e^{-3}$ & $3.8117 e^{-4}$ & $1.6056 e^{-4}$ & $5.4452 e^{-7}$ & 3.220 & 4.00 \\
& -0.9 & $9.8844 e^{-3}$ & $6.5600 e^{-4}$ & $7.4371 e^{-5}$ & $9.4331 e^{-7}$ & 4.350 & 4.25 \\
\hline
\end{tabular}

Example 2. Consider the PDE with a source term $f(x, y, z)$,

$$
u_{x x}+u_{y y}+u_{z}+\ell^{2} u=f(x, y, z), 0<x, y, z<1
$$

with the analytic solution is

$$
u(x, y, z)=\frac{\left(1-e^{100(x-1)}\right)\left(1-e^{100(y-1)}\right)\left(1-e^{100(z-1)}\right)}{\left(1-e^{-100}\right)^{3}} .
$$

The source function is given by the analytic solution with the boundary layers along $x=1, y=1$ and $z=1$. Hence a non-uniform grid along both directions with accumulation near $x=1, y=1$ and $z=1$ is used by the following stretching formula

$$
\begin{aligned}
& x_{i}=\frac{i}{N_{x}}+\frac{\lambda}{\pi} \sin \left(\frac{\pi i}{N_{x}}\right), \\
& y_{j}=\frac{j}{N_{y}}+\frac{\lambda}{\pi} \sin \left(\frac{\pi j}{N_{y}}\right), \\
& z_{k}=\frac{k}{N_{z}}+\frac{\lambda}{\pi} \sin \left(\frac{\pi k}{N_{z}}\right) .
\end{aligned}
$$

When $\lambda$ is going closer to 1 , more grids are accumulated near $x=1, y=1$ When the grids size is $32^{2}$ and $\lambda=0.8$, the distribution of grids is given as Figure 3. Table 2 indicates the maximum absolute errors CPU timing and order of accuracy for different stretching parameter $\lambda$, for Example 2. The value of $\lambda$ changes from 0.0 to 0.9 . We observed that in non-uniform grids with increasing the stretching parameter $\lambda$, more and more grids are accumulating into the boundary layers, consequently more accurate results are obtained from two different methods. Also, the rate of convergence is continuously increases with the increase of $\lambda$. We observed that when $\lambda=0.8$, then considerably most accurate solution is obtained with the HOC scheme, but when $\lambda$ is increased to 0.9 , it leads to decrease in accuracy. Fig. 4, shows the depicts the contours of the exact solution in the plane for $y=0.688$. (a) Exact solution (b) by HOC on uniform grids, (c) computed solution by the CDS scheme on uniform grids, (d) solution by HOC on non-uniform grids with $\lambda=0.8$.
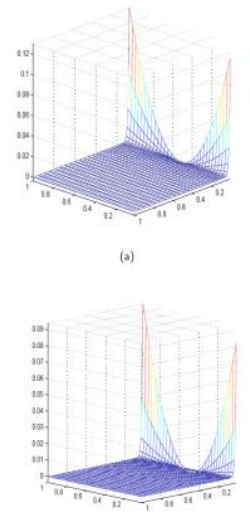

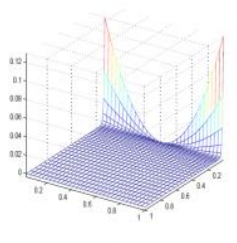

(b)

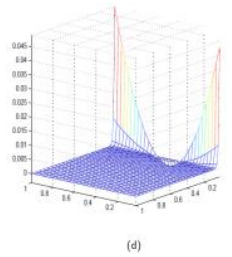

Figure 2: (a) Exact solution. (b) Computed solution from HOC scheme on uniform grids. (c) HOC scheme non-uniform grids. (d) CDS scheme with non-uniform grids. The error vector $e_{i j}=u_{i j}-v_{i j}$ and $N=32$ are the number of nodes and $k=10$ and $\lambda=-0.8$, for Example 1 .

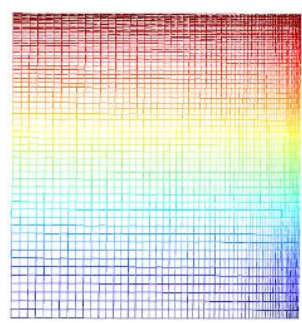

Figure 3: Non-uniform grids distribution in $x y$-plane, $3^{32}, \lambda=0.8$.

Table 2: Comparison of maximum absolute errors, CPU timing and order of accuracy of the two schemes for Example 2,

$\left\|e_{2}\right\|, \ell=10, N=16,32,64,128$

\begin{tabular}{|cc|c|c|c|c|c|c|}
\hline$N$ & $\lambda$ & $16^{2}$ & $32^{2}$ & $64^{2}$ & $128^{2}$ & CPU(seconds) & Order \\
\hline \multirow{4}{*}{ CDS } & 0.0 & $5.2002 e^{-2}$ & $4.8890 e^{-2}$ & $3.2290 e^{-2}$ & $7.1098 e^{-3}$ & 0.109 & 1.44 \\
& 0.2 & $3.4421 e^{-2}$ & $2.2213 e^{-2}$ & $1.1180 e^{-2}$ & $5.3112 e^{-3}$ & 0.155 & 1.82 \\
& 0.4 & $2.5541 e^{-2}$ & $1.7002 e^{-2}$ & $1.1040 e^{-2}$ & $3.1255 e^{-4}$ & 0.161 & 1.98 \\
& 0.6 & $1.1983 e^{-2}$ & $7.2100 e^{-3}$ & $4.1033 e^{-3}$ & $6.1043 e^{-5}$ & 0.880 & 2.44 \\
& 0.8 & $5.1287 e^{-3}$ & $1.9520 e^{-3}$ & $7.1530 e^{-5}$ & $1.6054 e^{-5}$ & 2.221 & 2.88 \\
& 0.9 & $2.4566 e^{-4}$ & $1.5424 e^{-4}$ & $7.8900 e^{-6}$ & $2.6041 e^{-6}$ & 2.233 & 3.87 \\
\hline \multirow{4}{*}{ HOC } & 0.0 & $4.2122 e^{-2}$ & $3.3211 e^{-3}$ & $7.4412 e^{-4}$ & $5.8234 e^{-4}$ & 0.142 & 1.52 \\
& 0.2 & $1.1218 e^{-2}$ & $1.6601 e^{-3}$ & $6.3318 e^{-4}$ & $4.7715 e^{-5}$ & 0.160 & 1.86 \\
& 0.4 & $9.7110 e^{-3}$ & $6.1218 e^{-4}$ & $3.6152 e^{-5}$ & $3.1961 e^{-6}$ & 0.416 & 2.00 \\
& 0.6 & $3.9328 e^{-4}$ & $4.1155 e^{-5}$ & $2.6001 e^{-6}$ & $2.3087 e^{-6}$ & 1.808 & 2.48 \\
& 0.8 & $3.7022 e^{-5}$ & $1.8676 e^{-6}$ & $5.2260 e^{-7}$ & $3.3350 e^{-7}$ & 3.541 & 2.96 \\
& 0.9 & $3.8844 e^{-5}$ & $2.8600 e^{-6}$ & $8.7210 e^{-7}$ & $5.3421 e^{-7}$ & 4.322 & 3.90 \\
\hline
\end{tabular}
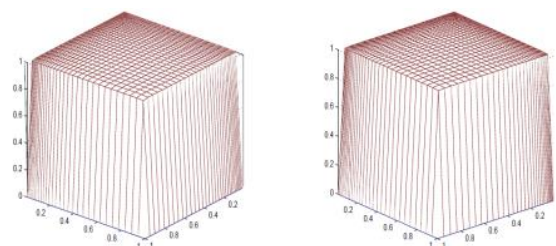

(a)

(b) 

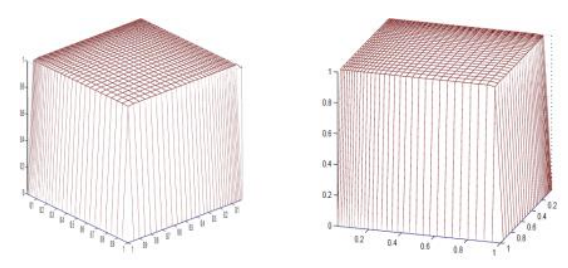

(d)

Figure 4: (a) Exact solution. (b) Computed solution from HOC scheme on uniform grids. (c) CDS scheme uniform grids. (d) HOC scheme on nonuniform grids, $3^{32}, \lambda=0.8$.

\section{CONCLUSION}

We have studied the Cauchy problem for Helmholtz type equation in two and three-dimensional cases. To deal with the instabilities of the solution of concerned problem a multigrid method is applied. The main advantage of multigrid method is that it provides accurate, stable, convergent numerical solution to the Cauchy type problem associated with Helmholtz equation $l=\Delta+k^{2}$ for $k \in \mathfrak{R}$ aid $k \in C$. We have used special multigrid methods which solve the resulting system efficiently. It is observed that multigrid method with the Gauss-Seidel relaxation work very well in solving the high-order scheme discretized 3D Helmholtz equation. The HOC scheme has three to fourth order accuracy and is more efficient than CDS scheme. It is also observed that on uniform grids the HOC and CDS schemes can attain its maximum accuracy. But in case of boundary layer problems with suitable grid stretching ratios, the HOC scheme has the desired accuracy. Therefore, it is reveal that grid distributions have much more significant effect on calculated accuracy for boundary layers problems. Numerical results show that multigrid method with HOC has the required accuracy by the accumulating many more grid points into the boundary layer and faster than the CDS scheme.

\section{REFERENCES}

[1] Beskos, D. 1997. Boundary element method in dynamic analysis, Part II (1986-1996). Applied Mechanics Reviews ASME, 50, 149-197.

[2] Harari, I., Barbone, P.E., Slavutin, M., Shalom, R. 1998. Boundary infinite elements for the Helmholtz equation in exterior domains. International Journal for Numerical Methods in Engineering, 41, 1105-1131.

[3] Hall, W.S., Mao, X.Q. 1995. A boundary element investigation of irregular frequencies in electromagnetic scattering. Engineering Analysis with Boundary Elements, 16, 245-252.

[4] Kern, D.Q., Kraus, A.D. 1972. Extended surface heat transfer. New York: McGraw-Hill.

[5] Manzoor, M., Ingham, D.B., Heggs. P.J. 1983. The one-dimensional analysis of fin assembly heat transfer. Journal of Heat Transfer ASME DC, $105,646-451$.

[6] Wood, A., Tupholme, G.E., Bhatti, M.I.H., Heggs, P.J. 1995. Steady-state heat transfers through extended plane surfaces. International Communications in Heat and Mass Transfer, 22, 99-109.

[7] Hadamard, J. 1923. Lectures on Cauchy problem in linear partial differential equations. London: University Press.

[8] Boisvert, R. F. 1987. A fourth-order accurate Fourier method for the Helmholtz equation in three dimensions. ACM Transactions on Mathematical Software, 13, 221-234.

[9] Sutmann, G. 1987. Compact finite difference scheme of sixth order for the Helmholtz equation. Journal of Computational and Applied Mathematics, 293, 15-31.

[10] Singer, I., Turkel, E. 1998. High-order_nite difference method for the Helmholtz equation. Computer Methods in Applied Mechanics and Engineering, 163, 343-358.
[11] Harari, I., and Turkel, E. 1995. Accurate _nite difference methods for time-harmonic wave propagation. Journal of Computational Physics, 119, 252-270.

[12] Harari, I., and Hughes, T. J. R. 1991. Finite element methods for the Helmholtz equation in an exterior domain model problem. Computer Methods in Applied Mechanics and Engineering, 87, 59-96.

[13] Mehdizadeh, O., Paraschiviou, M. 2003. Investigation of a twodimensional spectral method for Helmholtz equation. Journal of Computational Physics, 189, 111-129.

[14] Nabavi, M., Siddique, M. K., Dargahi, J. 2007. Sixth-order accurate compact finite-difference method for the Helmholtz equation. Journal of Sound and Vibration, 307, 972-982.

[15] Brandt, A. 1977. Multi-level adaptive solution to boundary value problems technique. Mathematics of Computation, 31, 333-390.

[16] Gupta, M. M., Kouatchou, J., and Zhang, J. 1997. Comparision of second and fourth order discretizations for multigrid Poisson solver. Journal of Computational Physics, 132, 226-232.

[17] Ghaffar, F., Islam, S., and Badshah, N. 2015. Multigrid method based on transformation free higher order scheme for solving 3D Helmholtz equation on nonuniform grids. Journal of Applied Environmental and Biological Sciences, 5, 85-97.

[18] Ghaffar, F., Badshah, N., and Islam, S. 2014. Multigrid Method for Solution of 3D Helmholtz Equation Based on HOC Schemes. Abstract and Applied Analysis, 1-15.

[19] Ghaffar, F., Badshah, N., Khan, M. A., and Islam, S. 2014. Multigrid method for 2D Helmholtz equation using higher order finite diffence scheme accelerated by Krylove subspace. Journal of Applied Environmental and Biological Sciences, 4, 169-179.

[20] Gupta, M. M., Kouatchou, J., and Zhang, J. 1997. A compact multigrid solver for convection-diffusion equation. Journal of Computational Physics, $132,123-129$.

[21] Ge, Y. and Cao, F. 2011. Multigrid method based on the transformationfree HOC scheme on non-uniform grids for 2D convection diffusion problems. Journal of Computational Physics, 230, 4051-4070.

[22] Kalnay De Rivas, E. 1972. On the use of nonuniform grids in finitedifference equations. Journal of Computational Physics, 10, 202-210.

[23] Teilgland, R., Eliassen, J. K. 2001. A multilevel mesh refinement procedure for CFD computations. International Journal for Numerical Methods in Fluids, 36, 519--538.

[24] Zhang, J., Sun, H., and Zhao, J. J. 2002. High order compact scheme with multigrid local mesh refinement procedure for convection-diffusion problems. Computer Methods in Applied Mechanics and Engineering, 191, 4661-4674.

[25] Ge, Y., and Cao, F. 2013. A transformation-free HOC scheme and multigrid method for solving the 3D Poisson equation on nonuniform grids. Journal of Computational Physics, 234, 199-216.

[26] Gryazin, Y. 2014. Preconditioned Krylov subspace methods for sixth order compact approximations of the Helmholtz equation, ISRN. Computers and Mathematics with Applications, 1, 1-15.

[27] Ge, Y. 2010. Multigrid method and fourth-order compact difference discretization scheme with unequal meshsizes for 3D Poisson equation. Journal of Computational Physics, 229, 6381-6391.

[28] Zhang, J. 1998. Fast and high accuracy multigrid solution for 3D Poisson equation. Journal of Computational Physics, 143, 449-461.

[29] Ghaffar, F., Badshah, N., Islam, S. and Khan, M. A. 2016. Multigrid method based on transformation-free high-order scheme for solving 2D Helmholtz equation on nonuniform grids. Advances in Difference Equations, (DOI 10.1186/s13662-016-0745-2), 1-16. 